\title{
Effect of Lifestyle on Periodontal Health Status in Jeddah, Saudi Arabia
}

\author{
Prathibha Anand Nayak ${ }^{1}$, Ullal Anand Nayak², Othman Wali3 ${ }^{3}$ Kavitha Odathurai Marusamy4, Nagihan K. Muhcu ${ }^{5}$ \\ 1,3,5 Department of Oral Basic \& Clinical Sciences, Ibn Sina National College for Medical Studies, Jeddah, Saudi Arabia. \\ 2, 4, Department of Preventive Dental Sciences, Ibn Sina National College for Medical Studies, Jeddah, Saudi Arabia.
}

\section{ABSTRACT}

\section{BACKGROUND}

The concept of a healthy lifestyle is reported to be directly related to health. Prevalence and severity of periodontal disease vary among different populations and regions which may be attributed to various lifestyle factors. The aim of the present study was to find the association between the various lifestyle factors and periodontal health.

\section{METHODS}

The present cross-sectional study was conducted among 374 participants aged 20 50 years during the 6 months period using a structured and pre-validated questionnaire. The lifestyle factors of selected participants were evaluated using an eight-item health practice index (HPI) scale given by Hagihara et al. Their clinical periodontal status was recorded by means of clinical attachment level using University of North Carolina (UNC)-15 probe. The statistical analysis was based on the association of components of lifestyle with severity of periodontitis was evaluated using chi square test. The multivariate regression analysis was used to evaluate the independent association of variables of life style, oral health care and health practice index with severity of periodontitis.

\section{RESULTS}

The statistical analysis revealed that severity of periodontitis increased with increasing age and decreased with increasing education level, income level, and frequency of dental visits and use of oral hygiene aids. The overall life style of participants did not have any significant association with the severity of periodontitis when multivariate logistic regression analysis was done.

\section{CONCLUSIONS}

There is a strong association of age, education, occupation, income, brushing device or method used, visit to the dentist, breakfast, and work hours per day with periodontal health.

\section{KEY WORDS}

Lifestyle, Periodontal Status, Health Practice Index
Corresponding Author: Dr. Ullal Anand Nayak, Associate Professor, Department of Preventive Dental Sciences, Ibn Sina National College for Medical Studies, Jeddah, Saudi Arabia. E-mail: pediatricdentist4u@gmail.com

DOI: $10.14260 /$ jemds/2021/164

How to Cite This Article: Nayak PA, Nayak UA, Wali O, et al. Effect of lifestyle on periodontal health status in Jeddah, Saudi Arabia. J Evolution Med Dent Sci 2021;10(11):760-767, DOI: $10.14260 /$ jemds/2021/164

Submission 19-11-2020,

Peer Review 23-01-2021, Acceptance 30-01-2021, Published 15-03-2021.

Copyright (C) 2021 Prathibha Anand Nayak et al. This is an open access article distributed under Creative Commons Attribution License [Attribution 4.0 International (CC BY 4.0)] 


\section{BACKGROUND}

Periodontal disease affects majority of the adult population and apparently poses public health concern. Although it is a plaque-induced disease, immunology, genetics, environmental and behavioural factors play a significant role in its aetiology and progression. It can lead to destruction of the periodontal ligament and its adjacent alveolar bone causing clinical attachment loss (CAL). The degree of clinical attachment loss is a measure of the severity of periodontal disease. ${ }^{1}$

Prevalence and severity of periodontal disease vary among different populations and regions which may be attributed to various factors. ${ }^{2}$ In recent times, lifestyle is gaining importance in the maintenance of periodontal health. Lifestyle has been defined as any activity undertaken by an individual and can include health behaviours and risk behaviours. It simply reflects the way people live, their attitudes and activities. It is acquired over the years through interaction with parents, siblings, teachers, friends and the environment they live, and may have direct or indirect effect on the progression of periodontal disease. ${ }^{3}$

An individual lifestyle assessment is usually used to selfreport behaviours such as eating habits, smoking and drinking habits, nutrition, sleep patterns, leisure-time activities, and physical activity. ${ }^{4}$ Although the relationship between lifestyle and health status has been studied before, their assessment scales are varied or complex. ${ }^{5}$

A strong relationship between seven lifestyle practices and physical health status was first documented by Belloc and Breslow (1972). ${ }^{6}$ Hagihara et al. modified the list of seven personal health practices mentioned above to construct a group of eight for use in a study in Japan. These eight were chosen as question items concerning personal health practices for the present study, and comprised items in a health practice index (HPI). ${ }^{7}$

The concept of a healthy lifestyle is reported to be directly related to health. ${ }^{8}$ They also found that people with an active lifestyle had fewer symptoms in teeth and gums.

Not all lifestyle factors are harmful and some of them can actually promote good oral health such as balanced diet, sufficient sleep, exercise etc.

These lifestyle behaviours are also associated with dental health which can be further understood and analysed. Although there are many studies associating certain lifestyle factors with general health, few studies are available that describe the influence of lifestyle factors on periodontal health in Saudi Arabia. Hence the present study was conducted to find the association between the various life style factors and periodontal health among the patients reporting to the dental clinics.

\section{METHODS}

The present cross-sectional study was conducted on the patients who reported during the period 1st September 2018 to $31^{\text {st }}$ March 2019 to Ibn Sina Dental Clinics, Jeddah, Saudi Arabia, after obtaining the ethical committee approval from the institution.
A structured questionnaire was prepared in English and pre-validated by three subject experts. The changes were done accordingly and translated to Arabic language by a subject expert. The questionnaire included demographic data, questions regarding oral health related habits and behaviour, lifestyle which consisted of four parts. The demographic data included participants' age, gender, location, marital status, income per month, education, occupation and religion.

The questions related to oral health behaviour included frequency of dental visits, device used for cleaning, cleaning method and frequency, habits such as tobacco and / or pan chewing.

The questionnaire was distributed to participants who gave their willingness to participate in the study in a consent form. The subjects who were selected belonged to 20 - 50 year age group, who were able to read either English or Arabic. Subjects not willing to participate, medically compromised, on medications or suffering from medical conditions affecting periodontal health, those undergoing orthodontic treatment, and using antibiotics, anti-inflammatory drugs or vitamin $\mathrm{C}$ supplements for the past 6 months were excluded from the study.

In order to detect a difference of $10 \%$, anticipating $70 \%$ of the subjects were of poor lifestyle individuals who had severe periodontal disease with a power of $80 \%$ at $95 \%$ confidence level, a minimum of 133 participants were needed to be enrolled in the study.

The lifestyle factors of selected participants were evaluated using an eight-item health practice index (HPI) scale given by Hagihara et $\mathrm{al}^{7}$ which included information regarding breakfast, diet, physical exercise, smoking, alcohol consumption, hours of work / day, hours of sleep / night and mental stress.

Subjects had to choose one out of $2-6$ available options for each item, and the answer was classified as indicating either "good" or "poor" health practices. The "good" health practices were coded 1 and "poor" as 0 . Each participant was then assigned a total score between 0 and 8 based on number of health practices and classified accordingly as having poor lifestyles (score 0 to 3), moderate lifestyles (scores 4 and 5), and good lifestyle (scores 6 to 8).

The clinical periodontal health status of participants was recorded by means of clinical attachment level using UNC-15 probe from the cement-enamel junction to the base of sulcus / pocket. ${ }^{9}$ Based on CAL, participants were classified as having gingivitis $(\mathrm{CAL}=0)$, mild periodontitis (mean $\mathrm{CAL}<2.5 \mathrm{~mm}$ ), moderate periodontitis (mean CAL 2.5 - $3.9 \mathrm{~mm}$ ) and severe periodontitis (mean CAL $\geq 4 \mathrm{~mm}$ ). ${ }^{10}$

A single trained examiner performed the clinical examination. Data collected from the questionnaire and the clinical examination were transferred to the Microsoft excel sheet and statistically analysed using International Business Machines Statistical Package for the Social Sciences (IBM SPSS) version 20. The association of components of lifestyle with severity of periodontitis was evaluated using chi square test. The multivariate logistic regression analysis was used to evaluate the independent association of variables of life style, oral health care and health practice index with severity of periodontitis. The level of significance chosen for all the comparisons was $\mathrm{p}<0.05$. 


\section{RESULTS}

\begin{tabular}{|ccc|}
\hline Variables & & $\mathbf{N}(\%)$ \\
Age & $20-35$ & $234(62.6 \%)$ \\
& $36-50$ & $140(37.4 \%)$ \\
Gender & Female & $193(51.6 \%)$ \\
& Male & $181(48.4 \%)$ \\
Marital status & Married & $173(46.3 \%)$ \\
& Unmarried & $201(53.7 \%)$ \\
Occupation & Unemployed & $142(38 \%)$ \\
& Unskilled & $67(17.9 \%)$ \\
& Clerical, farmer, business & $52(13.9 \%)$ \\
& Semi-skilled semi- & $113(30.2 \%)$ \\
Education & professional / professional & $259(69.3 \%)$ \\
& Primary, middle, high school & 259 \\
& Pre-university, diploma & $53(14.2 \%)$ \\
& Graduate, post graduate & $62(16.60 \%)$ \\
Income per month (in Riyals) & $\leq 2000$ & $38(10.2 \%)$ \\
& $2000-4000$ & $224(59.9 \%)$ \\
& 5000 - 9000 & $70(18.7 \%)$ \\
& $\geq 10000$ & $42(11.2 \%)$ \\
Geographic location & Urban & $284(75.9 \%)$ \\
& Rural & $90(24.1 \%)$ \\
\hline Table 1. Distribution of Study Population & \\
According to the Demographic Characteristics
\end{tabular}

\begin{tabular}{|c|c|c|c|}
\hline Lifestyle ( HPI) & $\begin{array}{c}\text { Participants } \\
\text { N = 374 } \\
\text { N (\%) }\end{array}$ & $\begin{array}{c}\text { Males } \\
\mathrm{N}=181 \\
\mathrm{~N}(\%)\end{array}$ & $\begin{array}{c}\text { Females } \\
\mathrm{N}=193 \\
\mathrm{~N}(\%)\end{array}$ \\
\hline Poor & 147 (39.3\%) & $77(42.5 \%)$ & $70(36.3 \%)$ \\
\hline 2. Moderate & $193(51.6 \%)$ & $85(47 \%)$ & 108 (56\%) \\
\hline 3. Good & $34(9.1 \%)$ & $19(40.5 \%)$ & $15(7.8 \%)$ \\
\hline $\begin{array}{r}\text { Table 2. Overall L } \\
\text { of the Study Pa } \\
\text { Lifestyles (Sc }\end{array}$ & $\begin{array}{l}\text { tyles (Combin } \\
\text { pants Poor Li } \\
=4,5) \text { Good I }\end{array}$ & $\begin{array}{l}\text { Health Pra } \\
\text { tyles (Score } \\
\text { estyle (Scort }\end{array}$ & $\begin{array}{l}\text { ndex Variab } \\
\text { 3) Moderat } \\
\text { or Higher) }\end{array}$ \\
\hline
\end{tabular}

374 participants were selected for the study from a total of 432 respondents, based on inclusion and exclusion criteria. The distribution of study population according to the demographic characteristics such as age, gender, marital status, occupation, education, income per month and geographic location is described in table 1.

The overall lifestyle of the population studied is shown in table 2. Table 3 describes the distribution of study participants according to the health practice index.

The relation of various components of life style was compared with the severity of periodontitis [Table 4]. It was observed that the factors such as age, gender, marital status, location, education, occupation, brushing device and method used, visit to the dentist, breakfast, exercise, sleep and work hours per day, smoking and life style have shown significant association with the severity of periodontitis [p $<0.05]$.

The independent association of lifestyle related demographic variables with severity of periodontitis using multivariate logistic regression analysis is shown in Table 6.

Although, the periodontitis was more severe in males compared to females [ $p=0.237]$; in married compared to unmarried [ $\mathrm{p}=0.140]$; and in rural compared to urban [ $\mathrm{p}=$ $0.752]$, it was observed that the periodontitis was significantly more severe in 36 to 50 year age group than 20 to 35 year age group [p $<0.001]$. It was significantly more severe [4.977 times] in individuals who have attained education level of primary schooling when compared to a graduate / postgraduate. Unemployed participants demonstrated significantly less severe [87 \% less] periodontitis when compared to semiskilled / semi-professional participants [p =
0.003]. Those earning less than 2000 SR exhibited 5.7 times more periodontitis than those earning more than 10000 SR per month [ $p=0.037]$.

\begin{tabular}{|c|c|c|}
\hline & Variables & $\begin{array}{c}\text { Frequency \& } \\
\text { Percentage }\end{array}$ \\
\hline \multirow{3}{*}{ Smoking } & Present & $113(30.2 \%)$ \\
\hline & Past & $45(12 \%)$ \\
\hline & Never & $216(57.8 \%)$ \\
\hline \multirow{2}{*}{ Alcohol consumption } & Yes & $0(0)$ \\
\hline & No & $374(100 \%)$ \\
\hline \multirow{3}{*}{ Breakfast } & Everyday & $166(44.4 \%)$ \\
\hline & Sometimes & $182(48.7 \%)$ \\
\hline & Never & $26(7 \%)$ \\
\hline \multirow{5}{*}{ Sleep hours at night } & $\geq 9 \mathrm{~h}$ & $54(14.4 \%)$ \\
\hline & $8 \mathrm{~h}$ & $99(26.5 \%)$ \\
\hline & $7 \mathrm{~h}$ & $74(19.8 \%)$ \\
\hline & $6 \mathrm{~h}$ & $107(28.6 \%)$ \\
\hline & $\leq 5 \mathrm{~h}$ & $40(10.7 \%)$ \\
\hline \multirow{5}{*}{ Work hours per day } & $\geq 11 \mathrm{~h}$ & $63(16.8 \%)$ \\
\hline & $10 \mathrm{~h}$ & $46(12.3 \%)$ \\
\hline & $9 \mathrm{~h}$ & $56(15 \%)$ \\
\hline & $8 \mathrm{~h}$ & $81(21.7 \%)$ \\
\hline & $\leq 7 \mathrm{~h}$ & $128(34.2 \%)$ \\
\hline \multirow{5}{*}{$\begin{array}{c}\text { Exercise } \\
\text { (frequency) }\end{array}$} & Almost everyday & $44(11.8 \%)$ \\
\hline & $\begin{array}{l}\text { About } 2 \text { to } 4 \text { days } \\
\text { per week }\end{array}$ & $53(14.2 \%)$ \\
\hline & About 1 day per week & $57(15.2 \%)$ \\
\hline & About 1 day per month & $39(10.4 \%)$ \\
\hline & Does not & $181(48.4 \%)$ \\
\hline \multirow{3}{*}{ Diet } & Eat a balanced diet & $71(19 \%)$ \\
\hline & Eat with little attention & $117(31.3 \%)$ \\
\hline & Don't eat a balanced diet & $186(49.7 \%)$ \\
\hline \multirow{3}{*}{ Stress } & Feeling excessive stress & $70(18.7 \%)$ \\
\hline & Feeling mild stress & $214(57.2 \%)$ \\
\hline & No stress & $90(24.1 \%)$ \\
\hline \multicolumn{3}{|c|}{$\begin{array}{c}\text { Table 3. Distribution of Study Participants } \\
\text { According to Health Practice Index }\end{array}$} \\
\hline
\end{tabular}

Table 5 shows the independent association of oral health care variables with severity of periodontitis using multivariate logistic regression analysis. The use of miswak or tooth brush alone was associated with increased severity of periodontitis when compared to those using tooth brush with interdental aids $[p<0.001]$. The severity of periodontitis was highest in those who performed vertical method of brushing [9.17 times] followed by circular method [6.39 times] and horizontal method [2.44 times]. Those who performed combination method had least severity when compared to all the other techniques and the difference was statistically significant.

Those participants who never visited dentists had significantly more severe periodontitis [3.32 times] when compared to those who visited once in 6 months [ $p=0.009]$.

Independent association of health practice index variables with severity of periodontitis was compared using multivariate logistic regression analysis in table 7. There were no significant associations between the severity of periodontitis with the diet, exercise, sleeping hours, smoking and stress levels.

Those who never had breakfast had more severe periodontitis when compared to those who had breakfast everyday $[p=0.010]$. Those who had work hours more than 11 hours per day had significantly lesser severity of periodontitis [77 \% less] when compared to those who had less than 7 hours of work per day. The overall lifestyle of participants did not have any significant association with the severity of periodontitis. 


\begin{tabular}{|c|c|c|c|c|c|c|c|}
\hline \multirow{2}{*}{\multicolumn{2}{|c|}{ Variables }} & \multicolumn{4}{|c|}{ Severity of Periodontitis } & \multirow{2}{*}{$\mathrm{X}^{2}$-Value } & \multirow{2}{*}{ P Value } \\
\hline & & \multirow{2}{*}{$\begin{array}{c}\text { Gingivitis } \\
19(8.1 \%) \\
0(0)\end{array}$} & \multirow{2}{*}{$\begin{array}{c}\text { Mild } \\
200(85.5 \%) \\
96(68.6 \%)\end{array}$} & \multirow{2}{*}{$\begin{array}{c}\text { Moderate } \\
12(5.1 \%) \\
34(24.3 \%)\end{array}$} & \multirow{2}{*}{$\begin{array}{l}\text { Severe } \\
03(1.3 \%) \\
10(7.1 \%)\end{array}$} & & \\
\hline Age & $\begin{array}{l}20-35 \\
36-50\end{array}$ & & & & & 49.321 & $<0.001$ \\
\hline \multirow{2}{*}{ Gender } & Male $(\mathrm{n}=181)$ & $01(0.6 \%)$ & $151(83.4 \%)$ & $23(12.7 \%)$ & $06(3.3 \%)$ & & \\
\hline & Female $(n=193)$ & $18(9.3 \%)$ & $145(75.1 \%)$ & $23(11.9 \%)$ & $07(3.6 \%)$ & 15.040 & 0.002 \\
\hline & Married & $0(0)$ & $128(74 \%)$ & $35(20.2 \%)$ & $10(5.8 \%)$ & & \\
\hline Marital status & Unmarried & $19(9.5 \%)$ & $168(83.6 \%)$ & $11(5.5 \%)$ & $03(1.5 \%)$ & 38.818 & $<0.001$ \\
\hline Jocation & Rural & $0(0)$ & $78(86.7 \%)$ & $08(8.9 \%)$ & $04(4.4 \%)$ & & \\
\hline Location & Urban & $19(6.7 \%)$ & $218(76.6 \%)$ & $38(13.4 \%)$ & $09(3.2 \%)$ & 8.309 & 0.040 \\
\hline & Primary, middle, high school & $06(2.3 \%)$ & $209(80.7 \%)$ & $36(13.9 \%)$ & $08(3.1 \%)$ & & \\
\hline Education & Pre-university, diploma & $06(11.3 \%)$ & $37(69.8 \%)$ & $06(11.3 \%)$ & $04(7.5 \%)$ & 24.122 & 0.004 \\
\hline & Graduate, post-graduate & $07(11.3 \%)$ & $50(80.6 \%)$ & $04(6.5 \%)$ & $01(1.6 \%)$ & & \\
\hline & Unemployed & $04(2.8 \%)$ & $118(83.1 \%)$ & $14(9.9 \%)$ & $06(4.2 \%)$ & & \\
\hline & Unskilled & $02(3 \%)$ & $50(74.6 \%)$ & $10(14.9 \%)$ & $05(7.5 \%)$ & & \\
\hline Occupation & Clerical, farmer, business & $0(0)$ & $45(86.5 \%)$ & $07(13.5 \%)$ & $0(0)$ & 22.362 & 0.008 \\
\hline & Semi-skilled, semi-professional & $13(11.5 \%)$ & $83(73.5 \%)$ & $15(13.3 \%)$ & $02(1.8 \%)$ & & \\
\hline & $<2000$ & $0(0)$ & $29(76.3 \%)$ & $05(13.2 \%)$ & $04(10.5 \%)$ & & \\
\hline & $2000-4000$ & $07(3.1 \%)$ & $179(79.9 \%)$ & $30(13.4 \%)$ & $08(3.6 \%)$ & & \\
\hline Income per month (Riyals) & $5000-9000$ & $06(8.6 \%)$ & $53(75.7 \%)$ & $10(14.3 \%)$ & $01(1.4 \%)$ & 12.454 & 0.053 \\
\hline & $\geq 10000$ & $06(14.3 \%)$ & $35(83.3 \%)$ & $01(2.4 \%)$ & $0(0)$ & & \\
\hline & None & $0(0)$ & $07(100 \%)$ & $0(0)$ & $0(0)$ & & \\
\hline & Miswak & $01(4.3 \%)$ & $16(69.6 \%)$ & $04(17.4 \%)$ & $02(8.7 \%)$ & & \\
\hline Brushing device & Tooth brush & $10(3.3 \%)$ & $243(80.7 \%)$ & $37(12.3 \%)$ & $11(3.7 \%)$ & 23.848 & 0.005 \\
\hline & Tooth brush with interdental cleaning aid & $08(18.6 \%)$ & $30(69.8 \%)$ & $05(11.6 \%)$ & $0(0)$ & & \\
\hline & Horizontal & $06(4.1 \%)$ & $121(81.8 \%)$ & $18(12.2 \%)$ & $03(2 \%)$ & & \\
\hline & Vertical & $01(1.5 \%)$ & 46 (68.7 \%) & $17(25.4 \%)$ & $03(4.5 \%)$ & & \\
\hline Brushing method & Circular & $02(2.9 \%)$ & $51(73.9 \%)$ & $09(13 \%)$ & $07(10.1 \%)$ & 40.293 & $<0.001$ \\
\hline & Combination & $10(12 \%)$ & $71(85.5 \%)$ & $02(2.4 \%)$ & $0(0)$ & & \\
\hline & Never & $0(0)$ & $01(100 \%)$ & $0(0)$ & $0(0)$ & & \\
\hline & Once & $03(2.3 \%)$ & $109(83.2 \%)$ & $15(11.5 \%)$ & $04(3.1 \%)$ & & \\
\hline Brushing frequency & Twice & $16(8.1 \%)$ & 147 (74.2\%) & $27(13.6 \%)$ & $08(4 \%)$ & 9.345 & 0.406 \\
\hline & Thrice & $0(0)$ & 32 (86.5 \%) & $04(10.8 \%)$ & $01(2.7 \%)$ & & \\
\hline & Never & $02(2 \%)$ & $73(74.5 \%)$ & $19(19.4 \%)$ & $04(4.1 \%)$ & & \\
\hline Visit to dentist & Once a year & $11(5.6 \%)$ & 155 (78.7 \%) & $23(11.7 \%)$ & $08(4.1 \%)$ & 18.905 & 0.004 \\
\hline & Once in 6 months & $06(7.6 \%)$ & $68(86.1 \%)$ & $04(5.1 \%)$ & $01(1.3 \%)$ & & \\
\hline & Yes & $03(3.6 \%)$ & $65(77.4 \%)$ & $11(13.1 \%)$ & $05(6 \%)$ & & \\
\hline Tobacco / pan chewing & No & $16(5.5 \%)$ & $231(79.7 \%)$ & $35(12.1 \%)$ & $08(2.8 \%)$ & 2.495 & 0.476 \\
\hline & Yes & $0(0)$ & $0(0)$ & $0(0)$ & $0(0)$ & & Inadequate \\
\hline Alcohol & No & $19(5.1 \%)$ & $296(79.1 \%)$ & $46(12.3 \%)$ & $13(3.5 \%)$ & - & sample \\
\hline & Everyday & $10(6 \%)$ & $143(86.1 \%)$ & $12(7.2 \%)$ & $01(0.6 \%)$ & & \\
\hline Breakfast & Sometimes & $09(4.9 \%)$ & $132(72.5 \%)$ & $32(17.6 \%)$ & $09(4.9 \%)$ & 21.646 & 0.001 \\
\hline & Never & $0(0)$ & $21(80.8 \%)$ & $02(7.7 \%)$ & $03(11.5 \%)$ & & \\
\hline & Balanced & $04(5.6 \%)$ & $54(76.1 \%)$ & $09(12.7 \%)$ & $04(5.6 \%)$ & & \\
\hline Diet & Eat with little attention & $07(6 \%)$ & $94(80.3 \%)$ & $10(8.5 \%)$ & $06(5.1 \%)$ & 6.382 & 0.382 \\
\hline & Not balanced & $08(4.3 \%)$ & $148(79.6 \%)$ & $27(14.5 \%)$ & $03(1.6 \%)$ & & \\
\hline & Everyday & $0(0)$ & $35(79.5 \%)$ & $07(15.9 \%)$ & $02(4.5 \%)$ & & \\
\hline & 2 - 4 days / week & $06(11.3 \%)$ & $42(79.2 \%)$ & $05(9.4 \%)$ & $0(0)$ & & \\
\hline Exercise & 1 day / week & $09(15.8 \%)$ & $35(61.4 \%)$ & $10(17.5 \%)$ & $03(5.3 \%)$ & 34.418 & 0.001 \\
\hline & 1 day / month & $02(5.1 \%)$ & $33(84.6 \%)$ & $04(10.3 \%)$ & $0(0)$ & & \\
\hline & Never & $02(1.1 \%)$ & $151(83.4 \%)$ & $20(11 \%)$ & $08(4.4 \%)$ & & \\
\hline & $\geq 9 \mathrm{hrs}$. & $04(7.4 \%)$ & $42(77.8 \%)$ & $03(5.6 \%)$ & $05(9.3 \%)$ & & \\
\hline & $8 \mathrm{hrs}$. & $04(04 \%)$ & $84(84.8 \%)$ & $09(9.1 \%)$ & $02(2 \%)$ & & \\
\hline Sleep hours per day & $7 \mathrm{hrs}$. & $07(9.5 \%)$ & $53(71.6 \%)$ & $14(18.9 \%)$ & $0(0)$ & 24.060 & 0.02 \\
\hline & $6 \mathrm{hrs}$. & $4(3.7 \%)$ & $83(77.6 \%)$ & $14(13.1 \%)$ & $6(5.6 \%)$ & & \\
\hline & $\leq 5 \mathrm{hrs}$ & $0(0)$ & $34(85 \%)$ & $06(15 \%)$ & $0(0)$ & & \\
\hline & Present & $1(0.9 \%)$ & $92(81.4 \%)$ & $15(13.3 \%)$ & $05(4.4 \%)$ & & \\
\hline Smoking & Past & $0(0)$ & $40(88.9 \%)$ & $03(6.7 \%)$ & $02(4.4 \%)$ & 13.639 & 0.034 \\
\hline & Never & $18(8.3 \%)$ & $164(75.9 \%)$ & $28(13 \%)$ & $06(2.8 \%)$ & & \\
\hline & Excessive & $02(2.9 \%)$ & $63(90 \%)$ & $05(7.1 \%)$ & $0(0)$ & & \\
\hline Stress & Mild & $15(7 \%)$ & $157(73.4 \%)$ & $32(15 \%)$ & $10(4.7 \%)$ & 12.407 & 0.053 \\
\hline & No & $02(2.2 \%)$ & $76(84.4 \%)$ & $09(10 \%)$ & $03(3.3 \%)$ & & \\
\hline & $\geq 11 \mathrm{hrs}$ & $04(6.3 \%)$ & $54(85.7 \%)$ & $05(7.9 \%)$ & $0(0)$ & & \\
\hline & 10 hrs. & $06(13 \%)$ & $31(67.4 \%)$ & $08(17.4 \%)$ & $01(2.2 \%)$ & & \\
\hline Work hours per day & $9 \mathrm{hrs}$. & $05(8.9 \%)$ & $47(83.9 \%)$ & $04(7.1 \%)$ & $0(0)$ & 32.334 & 0.001 \\
\hline & $8 \mathrm{hrs}$. & $0(0)$ & $60(74.1 \%)$ & $13(16 \%)$ & $08(9.9 \%)$ & & \\
\hline & $\leq 7 \mathrm{hrs}$ & $04(3.1 \%)$ & $104(81.3 \%)$ & $16(12.5 \%)$ & $04(3.1 \%)$ & & \\
\hline & Poor & $04(2.7 \%)$ & $127(86.4 \%)$ & $10(6.8 \%)$ & $06(4.1 \%)$ & & \\
\hline Life style & Moderate & $15(7.8 \%)$ & $141(73.1 \%)$ & $31(16.1 \%)$ & $06(3.1 \%)$ & 14.265 & 0.027 \\
\hline & Good & $0(0)$ & $28(82.4 \%)$ & $05(14.7 \%)$ & $01(2.9 \%)$ & & \\
\hline
\end{tabular}

\begin{tabular}{|c|c|c|c|c|}
\hline & & Adjusted OR (95\% CI) & $95 \%$ CI & $\mathbf{P}$ \\
\hline \multirow{4}{*}{ Brushing device } & None & 2.310 & $0.884-6.034$ & 0.087 \\
\hline & Miswak & 15.179 & $4.612-49.962$ & $<0.001$ \\
\hline & Toothbrush & 13.466 & $3.950-45.908$ & $<0.001$ \\
\hline & Toothbrush with interdental cleaning aid & 1 & & \\
\hline \multirow{4}{*}{ Brushing method } & Horizontal & 2.44 & $1.03-5.81$ & 0.043 \\
\hline & Vertical & 9.17 & $3.30-25.45$ & 0.000 \\
\hline & Circular & 6.39 & $2.30-17.73$ & 0.000 \\
\hline & Combination & 1.00 & & \\
\hline \multirow{3}{*}{ Visit to dentist } & Never & 3.32 & $1.36-8.11$ & 0.009 \\
\hline & Once a year & 1.41 & $0.65-3.04$ & 0.387 \\
\hline & Once in 6 months & 1.00 & & \\
\hline
\end{tabular}




\begin{tabular}{|c|c|c|c|c|}
\hline & bles & $\begin{array}{c}\text { Adjusted OR } \\
(95 \% \mathrm{CI})\end{array}$ & $95 \%$ CI & $\mathbf{P}$ \\
\hline Age & $\begin{array}{l}20-35 \\
36-50\end{array}$ & $\begin{array}{c}0.089 \\
1\end{array}$ & $0.032-0.249$ & $<0.001$ \\
\hline Gender & $\begin{array}{l}\text { Male } \\
\text { Female }\end{array}$ & $\begin{array}{c}1 \\
0.588\end{array}$ & $0.244-1.418$ & 0.237 \\
\hline Marital status & $\begin{array}{l}\text { Married } \\
\text { Unmarried }\end{array}$ & $\begin{array}{c}2.065 \\
1\end{array}$ & $0.789-5.404$ & 0.140 \\
\hline Location & $\begin{array}{l}\text { Rural } \\
\text { Urban }\end{array}$ & $\begin{array}{c}1.130 \\
1\end{array}$ & $0.531-2.405$ & 0.752 \\
\hline Education & $\begin{array}{c}\text { Primary, middle, high school } \\
\text { Pre-university, diploma } \\
\text { Graduate, Post-graduate }\end{array}$ & $\begin{array}{c}4.977 \\
2.503 \\
1\end{array}$ & $\begin{array}{c}1.496-16.553 \\
0.76-8.515\end{array}$ & $\begin{array}{l}0.009 \\
0.142\end{array}$ \\
\hline Occupation & $\begin{array}{c}\text { Unemployed } \\
\text { Unskilled } \\
\text { Clerical, farmer, business } \\
\text { Semi-skilled, semi-professional }\end{array}$ & $\begin{array}{c}0.137 \\
0.396 \\
0.467 \\
1\end{array}$ & $\begin{array}{l}0.037-0.504 \\
0.121-1.296 \\
0.146-1.490\end{array}$ & $\begin{array}{l}0.003 \\
0.126 \\
0.198\end{array}$ \\
\hline Income per months(in Riyals) & $\begin{array}{c}<2000 \\
2000-4000 \\
5000-9000 \\
\geq 10000\end{array}$ & $\begin{array}{c}5.711 \\
1.868 \\
0.558 \\
1\end{array}$ & $\begin{array}{c}1.109-29.408 \\
0.518-6.742 \\
0.143-2.181\end{array}$ & $\begin{array}{l}0.037 \\
0.340 \\
0.402\end{array}$ \\
\hline
\end{tabular}

\begin{tabular}{|c|c|c|c|c|}
\hline & Variables & $\begin{array}{c}\text { Adjusted OR } \\
\text { (95\% CI) }\end{array}$ & $95 \%$ CI & $\mathbf{P}$ \\
\hline \multirow[t]{3}{*}{ Breakfast } & Everyday & 0.22 & $0.07-0.70$ & 0.010 \\
\hline & Sometimes & 0.71 & $0.21-2.36$ & 0.572 \\
\hline & Never & 1.00 & & \\
\hline \multirow[t]{3}{*}{ Diet } & Eat a balanced diet & 0.894 & $0.367-2.176$ & 0.805 \\
\hline & Eat with little attention & 0.963 & $0.454-2.045$ & 0.922 \\
\hline & Don't eat a balanced diet & 1 & & \\
\hline \multirow[t]{5}{*}{ Exercise } & Almost everyday & 2.855 & $0.947-8.608$ & 0.062 \\
\hline & About 2 to 4 days per week & 0.638 & $0.189-2.148$ & 0.468 \\
\hline & About 1 day per week & 0.391 & $0.129-1.192$ & 0.099 \\
\hline & About 1 day per month & 0.773 & $0.238-2.507$ & 0.668 \\
\hline & Does not & 1 & & \\
\hline \multirow[t]{5}{*}{ Sleeping hours } & $\geq 9$ hrs. & 2.609 & $0.668-10.197$ & 0.168 \\
\hline & $8 \mathrm{hrs.}$ & 0.684 & $0.198-2.364$ & 0.549 \\
\hline & $7 \mathrm{hrs}$. & 0.882 & $0.237-3.284$ & 0.851 \\
\hline & $6 \mathrm{hrs}$. & 0.649 & $0.204-2.063$ & 0.464 \\
\hline & $\leq 5 \mathrm{hrs}$. & 1 & & \\
\hline \multirow[t]{3}{*}{ Smoking } & Present & 1.8854 & $0.802-4.288$ & 0.149 \\
\hline & Past & 0.696 & $0.223-2.167$ & 0.531 \\
\hline & Never & 1 & & \\
\hline \multirow[t]{3}{*}{ Stress } & Feeling excessive stress & 1.747 & $0.607-5.027$ & 0.301 \\
\hline & Feeling mild stress & 1.249 & $0.505-3.090$ & 0.630 \\
\hline & No stress & 1 & & \\
\hline \multirow[t]{5}{*}{ Work hours } & $\geq 11$ hrs. & 0.233 & $0.077-0.708$ & 0.010 \\
\hline & $10 \mathrm{hrs}$. & 0.470 & $0.131-1.687$ & 0.247 \\
\hline & $9 \mathrm{hrs}$. & 0.231 & $0.069-0.778$ & 0.018 \\
\hline & $8 \mathrm{hrs}$. & 1.326 & $0.512-3.432$ & 0.561 \\
\hline & $\leq 7 \mathrm{hrs}$. & 1 & & \\
\hline \multirow[t]{3}{*}{ Life styles } & Poor & 0.70 & $0.21-2.37$ & 0.566 \\
\hline & Moderate & 0.42 & $0.14-1.25$ & 0.121 \\
\hline & Good & 1.00 & & \\
\hline
\end{tabular}

\section{DISCUSSION}

Periodontal diseases are linked to lifestyles and the associated risk factors may influence oral health habits and periodontal health. However, the culture and various lifestyle indicators tend to vary in different parts of the world. ${ }^{11}$ Also there are no standardized methods for measuring lifestyle at present. In the present study too, the life style has not shown significant association with periodontal disease. Hence, it becomes important to design a lifestyle variable using a numerous sets of behaviours which can do the task of health orientation better than those individual behaviour priorly used.

There is an increase in severity of periodontal diseases in the older age group, matching with the results of previous studies. The increased severity may be related more to an increase in the exposure time to causative agents rather than a damage rate increase in periodontal tissues. ${ }^{12}$ It has been observed that severity of periodontal diseases increase with the advancing age. The increasing severity with advancing age may be attributed to general deterioration in immune function and tissue integrity which increases the vulnerability of periodontal tissues to diseases. ${ }^{13}$

Females when compared to males tend to have better periodontal status and a more positive dental health behaviour. They seem to follow a healthier lifestyle due to their concern for aesthetics and attractive appearance. At the same time, they exhibit better knowledge and attitude towards general and oral health. ${ }^{14}$

Person et al. demonstrated a similar distribution of periodontitis among the married couples and also among married study participants whose spouses were not a part of the study. Thus periodontitis is likely to be present among both the partners or neither of them had it.15 Married couples share certain social factors and health habits placing them at either greater or lower risk for periodontal or systemic disease. 16 
A longitudinal study of 5 years found a significant association between developing periodontal disease and the participant's occupation. The multi-regression analysis suggested that periodontal disease risk and severity was greater for skilled workers, sales persons and drivers when it was compared to professionals. The skilled workers and drivers tend to have prolonged working hours, leading to lack of good quality of sleep and rest and higher levels of stress. ${ }^{17}$

A study conducted among 18 - 64 years old with similar socio-economic status reported various social determinants of health such as social security, low educational qualifications, utilization of dental care and behavioural practices like excessive tobacco consumption to be the major risk factors for periodontal diseases. ${ }^{18}$ Individuals with higher education and higher income, often live in better living conditions and are less likely to smoke. ${ }^{19}$

Another important social aspect to consider is religiosity which may be a protective factor against periodontal diseases through extrinsic factors, by higher social support, and intrinsic factors, by higher spirituality pathways. ${ }^{20}$ In the present study, all participants were of the same religion.

It has been observed that people visit the dentist mainly for their treatment needs rather than disease prevention. People hailing from lower income group exhibit poorer dental health behaviour. Regular dental visit may be related to occupational status rather than the oral cleaning habits and females have been found to visit the dentist more frequently as compared to males. ${ }^{21}$ In the present study, the frequency of dental visit is inversely related to the severity of periodontitis.

In the present study, periodontal disease was observed to be more prevalent among the rural population compared to urban which was statistically not significant. A similar prevalence of periodontal disease was observed in urban \& rural areas in another study. ${ }^{22}$ People belonging to very low socioeconomic status residing in rural areas may have more severe periodontal disease due to inadequate dental facilities and poor oral health habits. Thus there is a necessity to improvise public health policy and include the dental professionals into health teams. ${ }^{23}$

Smoking reduces the blood supply and delays healing of periodontal tissues. It influences the quality and quantity of periodontal pathogens within the periodontal tissues, and affects host immune responses [low antibody synthesis, impaired phagocytosis and chemotactic function of polymorphonuclear neutrophils (PMN)].24 Although periodontal diseases and smoking are observed among middle-aged and older adults, their prevalence is gradually increasing among the teenagers and young adults. ${ }^{25}$ In the present study, smoking was associated with periodontal disease severity. Certain studies in Saudi Arabia also found association between cigar / pipe smoking and elevated levels of periodontal disease. ${ }^{26}$

Skipping of breakfast was commonly observed among the individuals with periodontitis ${ }^{27}$ which is in accordance with the results of present study. The other unhealthy dietary habits like consumption of less vegetables or fruits and frequent snacking habit can predispose to overweight and obesity further increasing the risk for periodontitis. ${ }^{28}$

Sleep deprivation affects various physiological functions related to memory, cognition, risk of depression and hormonal regulation. Acute as well as chronic sleep deprivation can activate inflammatory processes, increasing $\mathrm{C}$-reactive protein levels, increasing peripheral leukocyte circulation and increasing the levels of tumour necrosis factor-alpha \& interleukin-6. Since periodontitis and sleep deprivation have inflammation as a common characteristic, an association of sleep duration and periodontitis was observed with varying results. ${ }^{29}$

It has been found that engaging in long work hours or abnormal / busy schedules are related to deteriorating health conditions, and periodontitis may be one of the important signs. Long work hours can activate the hypothalamicpituitary-adrenal stress-response system, stimulating the adrenal cortex to release glucocorticoids and affecting the immune function. ${ }^{30}$ Work stress also stimulates adrenal medulla releasing catecholamines. Stress-related hormones play a role in periodontal disease progression as such patients show above-normal concentrations of salivary cortisol and urinary metanephrine which is a metabolite of adrenaline. ${ }^{31}$ The stress-response system may also attenuate immunity, leading to periodontal tissue breakdown. Due to inadequate recovery time from work stress which causes dysfunction of immune system, there is an increased chance for infectious agents to enter bloodstream and cause disease. Hence, those who work for long hours are more vulnerable for aggravation of periodontal disease. ${ }^{32}$

Habitual exercise result in reduced obesity and lifestyle related diseases by activating the immune system and reducing inflammatory cytokines. The same mechanism improves the periodontal status among those who exercise regularly. The effect may be similar to that of nonsurgical periodontal treatment performed on obese individuals. The recent literature thus suggests that periodontal treatment and exercise habits have a synergistic effect in the improvement of periodontal disease. ${ }^{33}$

Oral hygiene habits vary across the world and people of high socio-economic status tend to adopt healthier lifestyles, with regular tooth brushing and use of fluoridated tooth paste. ${ }^{34}$ The literature suggests that the important risk factors for poor oral health among the Kingdom of Saudi Arabia (KSA) population include different patterns of tobacco use, moderate utilization of dental health care facilities and lack of oral selfcare. However, the findings are general with limited evidence necessitating high quality studies to establish greater level of evidence in the region of KSA. ${ }^{35}$ In KSA, miswak is the traditional way of cleaning teeth and its use has been associated with a decreased need for periodontal disease management, although the rates of periodontitis were not quantified in the study. ${ }^{36} \mathrm{~A}$ thorough tooth brushing for 2 minutes has been found to be effective in maintaining good oral health.

Tooth brushing was associated with lower prevalence of periodontal disease when performed after lunch and before bedtime, and lower severity of periodontal disease was seen among those who brushed their teeth after dinner also. Further, the use of floss along with tooth brushing was associated with a lower prevalence of periodontal disease as compared to tooth brushing alone ${ }^{37}$ which was similar to the results of the present study. The use of inter-dental aids such as dental floss and toothpicks show wide variation with respect to personal income, level of education and place of residence. Among these, education level has been found to be a strong determinant of oral hygiene practices in the studies done in different countries. ${ }^{38}$ The use of manufactured 
toothbrushes, are gradually being adopted by the middle and low income countries; but, the use of fluoridated toothpaste is yet to be adopted.

Awareness of people regarding prevention of periodontal disease is varied and the relevance of tobacco and diet is seldom emphasized to the common man. Further, knowledge regarding the aetiology and prevention of periodontal disease is lower than that for dental caries. ${ }^{38}$ Therefore, a paradigm shift in treatment strategy that is population based rather than individual based should be introduced to promote periodontal health and to address social inequalities in periodontal health status. Determinants of periodontal diseases such as poor oral hygiene, excessive tobacco use, systemic diseases and psychosocial factors need to be focused. Although expensive and time consuming, few time-series studies have been conducted on periodontal health. This data would help to facilitate the prevention and early treatment of periodontal disease with an evidence-based approach.

\section{CONCLUSIONS}

The present study highlights a strong association between periodontal health and age, education, occupation, income, oral hygiene practices, visit to the dentist, breakfast and work hours per day.

The study participants represent a small proportion of population and hence there is a need for studies with larger sample size to establish their association. Further life style indicators that suit the culture and traditions followed in each country can be taken into account in future studies.

Data sharing statement provided by the authors is available with the full text of this article at jemds.com.

Financial or other competing interests: None.

Disclosure forms provided by the authors are available with the full text of this article at jemds.com.

\section{REFERENCES}

[1] Dye BA, Selwitz RH. The relationship between selected measures of periodontal status and demographic and behavioural risk factors. J Clin Periodontol 2005;32(7):798-808.

[2] Klinge B, Norlund A. A socio-economic perspective on periodontal diseases-a systematic review. J Clin Periodontol 2005;32(Suppl 6):314-25.

[3] Yoshida Y, Ogawa Y, Imaki M, et al. Lifestyles and periodontal disease of Japanese factory workers. Environ Health Prev Med 1997;1(4):188-92.

[4] Purohit B, Singh A. Lifestyle and oral health. Adv life Sci Technol 2012;3:35-43.

[5] Noguchi Y, Ueda K, Fukumoto K, et al. Relationship between perceived health status and health practices in the general adult population in Japan. Open J Prev Med 2015;5(6):280-9.

[6] Belloc NB, Breslow L. Relationship of physical health status and health practices. Prev Med 1972;1(3):409-21.

[7] Hagihara A, Morimoto K. Personal health practices and attitudes toward nonsmokers' legal rights in Japan. Soc Sci Med 1991;33(6):717-21.
[8] Wiley JA, Camacho TC. Lifestyle and future health: evidence from the Alameda county study. Prev Med 1980;9(1):1-21.

[9] Rheu GB, Ji S, Ryu JJ, et al. Risk assessment for clinical attachment loss of periodontal tissue in Korean adults. J Adv Prosthodont 2011;3(1):25-32.

[10] Torrungruang K, Tamsailom S, Rojanasomsith K, et al. Risk indicators of periodontal disease in older Thai adults. J Periodontol 2005;76(4):558-65.

[11] Abel T. Measuring health lifestyles in a comparative analysis: theoretical issues and empirical findings. Soc Sci Med 1991;32(8):899-908.

[12] Aljehani YA. Risk factors of periodontal disease: review of the literature. Int J Dent 2014;2014:182513.

[13] Behal R, Saima S, Jan SM. Prevalence of periodontal diseases in relation to associated risk factors/indicators amongst patients attending a government dental hospital in Kashmir. Int J Contemp Med Res 2016;3(10):3110-13.

[14] Gundala R, Chava VK. Effect of lifestyle, education and socioeconomic status on periodontal health. Contemp Clin Dent 2010;1(1):23-6.

[15] Persson GR, Persson RE, Hollender LG, et al. The impact of ethnicity, gender and marital status on periodontal and systemic health of older subjects in the trials to enhance elders' teeth and oral health (TEETH). J Periodontol 2004;75(6):817-23.

[16] Von Troil-Lindén B, Alaluusua S, Wolf J, et al. Periodontitis patient and the spouse: periodontal bacteria before and after treatment. J Clin Periodontol 1997;24(12):893-9.

[17] Sheiham A, Nicolau B. Evaluation of social and psychological factors in periodontitis. Periodontol 2000 2005;39:118-31.

[18] Singh A, Purohit BM, Masih N, et al. Risk factors for oral diseases among workers with and without dental insurance in a national social security scheme in India. Int Dent J 2014;64(2):89-95.

[19] Watson AC, Nilam S. Educational level as a social determinant of health and its relationship to periodontal disease as a health outcome. J Dent Sci Ther 2017;1(3):811.

[20] Zini A, Sgan-Cohen HD, Marcenes W. Is religiosity related to periodontal health among the adult Jewish population in Jerusalem? J Periodontal Res 2012;47(4):418-25.

[21] Sanders AE, Slade GD, Turrell G, et al. The shape of the socioeconomic-oral health gradient: implications for theoretical explanations. Community Dent Oral Epidemiol 2006;34(4):310-9.

[22] Singh GP, Soni BJ. Prevalence of periodontal diseases in urban and rural areas of Ludhiana, Punjab. Indian J Community Med 2005;30(4):127-9.

[23] de Macêdo TCN, Costa MDCN, Gomes-Filho IS, et al. Factors related to periodontal disease in a rural population. Braz Oral Res 2006;20(3):257-62.

[24] Palmer RM, Wilson RF, Hasan AS, et al. Mechanisms of action of environmental factors-tobacco smoking. J Clin Periodontol 2005;32(Suppl 6):180-95.

[25] Vered Y, Livny A, Zini A, et al. Periodontal health status and smoking among young adults. J Clin Periodontol 2008;35(9):768-72. 
[26] Albandar JM, Streckfus CF, Adesanya MR, et al. Cigar, pipe and cigarette smoking as risk factors for periodontal disease and tooth loss. J Periodontol 2000;71(12):187481.

[27] Tomofuji T, Furuta M, Ekuni D, et al. Relationships between eating habits and periodontal condition in university students. J Periodontol 2011;82(12):1642-9.

[28] Harada S, Akhter R, Kurita K, et al. Relationships between various aspects of lifestyle and dental health behaviors in a rural population in Japan. Community Dent Oral Epidemiol 2005;33(1):17-24.

[29] Wolk R, Gami AS, Garcia-Touchard A, et al. Sleep and cardiovascular disease. Curr Probl Cardiol 2005;30(12):625-62.

[30] Lee W, Lim SS, Kim B, et al. Relationship between long working hours and periodontitis among the Korean workers. Sci Rep 2017;7(1):7967.

[31] Mesa F, Magán-Fernández A, Muñoz R, et al. Catecholamine metabolites in urine, as chronic stress biomarkers, are associated with higher risk of chronic periodontitis in adults. J Periodontol 2014;85(12):175562.

[32] Geurts SAE, Sonnentag S. Recovery as an explanatory mechanism in the relation between acute stress reactions and chronic health impairment. Scand J Work Environ Health 2006;32(6):482-92.

[33] Omori S, Uchida F, Oh S, et al. Exercise habituation is effective for improvement of periodontal disease status: a prospective intervention study. Ther Clin Risk Manag 2018;14:565-74.

[34] Albertsson KW, van Dijken JWV. Awareness of toothbrushing and dentifrice habits in regularly dental care receiving adults. Swed Dent J 2010: 34(2):71-8.

[35] Alshammari AKS, Wahi MM. A narrative review of the prevalence of periodontitis in Saudi Arabia: a proposal for a national oral health research agenda for vision 2030. The Open Dent J 2019;13(1):171-6.

[36] Al-Khateeb TL, O'Mullane DM, Whelton $\mathrm{H}$, et al. Periodontal treatment needs among Saudi Arabian adults and their relationship to the use of the Miswak. Community Dent Health 1991;8(4):323-8.

[37] Han K, Park JB. Association between oral health behavior and periodontal disease among Korean adults: the Korea national health and nutrition examination survey. Medicine (Baltimore) 2017;96(7):e6176.

[38] Petersen PE. Inequalities in oral health: the social context for oral health. In: Pine C, Harris R, eds. Community Oral Health. London: Quintessence 2007: p. 31-58. 